\title{
Drug Resistance Pattern in the Clinical Isolates of Pulmonary Tuberculosis
}

\section{Apoorva Narain, Ajay Kumar Verma, Anand Srivastava and Surya Kant*}

Department of Respiratory Medicine, King George's Medical University, Lucknow, Uttar Pradesh, India

*Corresponding Author: Surya Kant, Professor and Head, Department of Respiratory Medicine, King George's Medical University, Uttar Pradesh, India E-mail: skantpulmed@gmail.com

Received: August 27, 2019; Published: September 16, 2019

DOI: $10.31080 /$ ASMI.2019.02.0374

\begin{abstract}
Multidrug-resistant tuberculosis (MDR-TB) is a major public health threat, especially in a country like India, which carries the highest global burden of tuberculosis (TB). TB is caused by Mycobacterium tuberculosis (MTB); the bacteria possess multiple mechanisms that provide it both intrinsic and acquired resistance to current anti-TB drugs. In the current treatment regimen followed for TB, rifampicin and isoniazid are the major drugs given in the first line of treatment. Fluoroquinolones and aminoglycosides are the second line drugs given in case the MTB is resistant to first line drugs. Acquired resistance is mainly due the exposure of MTB to suboptimal concentration of drugs, either due to inadequate treatment given by the medical practitioner or due to the discontinuous treatment regimen followed by patients. Treating drug susceptible TB requires a treatment regimen of almost 6 months which increases significantly from 9 months to 24 months in case of drug resistant cases. In the study conducted we determined the resistance patterns to first and second line anti-TB drugs and the frequency of MDR-TB. Contemplating the drug resistance pattern of MTB will help understanding the epidemiological parameters involved.
\end{abstract}

Keywords: Mycobacterium tuberculosis; Drug Resistance; Multi Drug Resistance

\section{Abbreviations}

TB: Tuberculosis; DR-TB: Drug Resistant Tuberculosis; HIV: Human Immunodeficiency Virus; MDR: Multi Drug resistant; XDR: Extensively Drug Resistant; MTB: Mycobacterium tuberculosis; PTB: Pulmonary Tuberculosis; EPTB: Extra Pulmonary TB; RIF: Rifampicin; INH: Isoniazid; PYZ: Pyrazinamide; EMB: Ethambutol AGs: Aminoglycosides; FQs: Fluoroquinolones; SM: Streptomycin; KM: Kanamycin; OFX: Ofloxacin; AM: Amikacin; WHO: World Health Organization; The Union: The International Union against Tuberculosis and Lung Disease; MTBC: MTB Complex; PDR: Poly Drug Resistance; KGMU: King George's Medical University; ZN: Ziehl Neelsen; LJ: Lowenstein-Jensen; DST: Drug Susceptibility Testing; NALC-NaOH: N-acetyl-L-cysteine-sodium citrate- sodium-hydroxide; IEC: Institutional Ethics Committee; RNTCP: Revised National Tuberculosis Control Programme.

\section{Introduction}

India carries the largest global burden of tuberculosis (TB).The currently prevailing scenarios of drug-resistant tuberculosis (DRTB) are particularly alarming, and pose a significant threat to the control of the disease globally $[1,2]$. There is also much concern that the TB situation has become worse due to Human Immunodeficiency Virus (HIV), as the viral infection weakens the host immune system and predispose to endogenous reactivation and exogenous re-infection with TB. DR-TB and HIV co-infection presents a serious challenge for effective TB control. The increasing emergence of multidrug-resistant (MDR) and extensively drug-resistant (XDR) TB presents another major threat to effectively control TB [3]. Drug resistance in Mycobacterium tuberculosis (MTB) arises from spontaneous chromosomal mutations at low frequency. Clinical DR-TB largely occurs as a result of selection during disease treatment of these genetic alterations through erratic drug supply, suboptimal physician prescription and poor patient adherence. Molecular mechanisms of DR have been elucidated for the major first- and second-line drugs Rifampicin (RIF), Isoniazid (INH), Pyrazinamide (PYZ), Ethambutol (EMB), the aminoglycosides (AGs) and the fluoroquinolones (FQs) [4-6]. The relationship between drug resistance in MTB strains and their virulence/transmissibility needs to be further investigated. Understanding the mechanisms of drug resistance in MTB would enable the development of rapid molecular diagnostic tools and furnish possible insights into new drug development for the treatment of TB [5]. 
In a recent report on the anti-tuberculosis drug resistance surveillance global project published by the World Health Organization (WHO) and the International Union against Tuberculosis and Lung Disease (The UNION), the proportion of MDR denoting resistance to at least RIF and INH in new cases ranged from $0 \%$ to $22.3 \%$. The highest proportion of MDR-TB reported among previously treated cases was $60 \%[7,8]$. The proportion of XDR-TB, defined as MDRTB with additional bacillary resistance to fluoroquinolones (FQs) and at least one second-line injectable drug in different settings, ranged from $0 \%$ to $30 \%$ globally. Around 40,000 cases of XDR-TB are estimated to emerge globally each year $[2,9,10]$.

The aim of this study was to review the current incidence and the pattern of drug resistance for TB patients. MDR- TB is $2.2 \%$ of the new cases and $38.4 \%$ of re-treatment cases (according to a national survey in 2002). The survey deduces the proportion of MDR-TB patients amongst newly and previously treated TB patients diagnosed at tertiary care hospital.

\section{Materials and Methods}

Study population

To start with 218 MTB clinical isolates were taken up for drug resistance study. All subjects recruited from Department of Respiratory Medicine, King George's Medical University (KGMU) UP, Lucknow, were newly diagnosed Pulmonary TB (PTB) patients registered under RNTCP attending OPD or admitted to the hospital from March 2017 to August 2018. All patients selected were HIV negative. The patients presented smear positive PTB, either newly diagnosed cases or cases with treatment failure. The patient groups were categorized into three types: 1) New cases, 2) Retreated cases, 3) Treatment failure cases. Only patients who provided voluntary written consent were recruited for the study.

\section{Inclusion criteria}

In our study, every case presented by clinical and radiological findings suggestive of PTB and sputum positive for mycobacterial TB by Ziehl Neelsen (ZN) technique, was collected; whether a new fresh case or previously treated case, regardless of their age, gender, occupation, other co-morbid conditions, receiving anti-mycobacterial treatment or not. Newly diagnosed sputum smear-positive pulmonary TB patients with no history of prior treatment for $\mathrm{TB}$, or a history of anti-TB treatment for less than 30 days and diagnosed patients with sputum smear-positive PTB with a history of previous TB episode with more than 30 days of anti-TB treatment. This may include relapses, treatment after default, treatment after failure, or other patients who have claimed to have anti-TB treatment for more than 30 days.
Patients with sputum smear-negative PTB; patients with extra pulmonary TB (EPTB) and patients unwilling to give informed consent were excluded. Patients were subjected to full history crossexamination, clinical examination, chest radiography, laboratory study, sputum examination by Ziehl Neelsen technique, culture using Lowenstein-Jensen (LJ) media and drug susceptibility testing (DST) using the proportion method [11].

\section{Collection of MTB Isolates}

All specimens were handled in a negative pressure environment as per the international standards for biosafety and infection control for MTB. For three consecutive mornings sputum samples from each patient were collected in properly labeled screw cap disposable plastic bottles and were decontaminated using N-acetylL-cysteine-sodium citrate- sodium-hydroxide (NALC-NaOH) procedure [12]. Sputum samples were processed and stained for Acid Fast Bacilli (AFB).

One sputum sample from each smear positive patient was inoculated on LJ slants and incubated in automated culture system at $37^{\circ} \mathrm{C}$ for four to six weeks. Preliminary identification of mycobacterial isolates depends on their growth on LJ slants. Specific identification was accomplished by the performance of ZN stain and battery of biochemical tests. The positive cultures include growth in LJ medium after decontamination of sputum samples and incubation at $37^{\circ} \mathrm{C}$ for $4-6$ weeks. MTB isolates were recovered from 207 HIV negative and smear positive cases of both genders, age varying from 18 to 60 years, with MDR-TB that was refractory to chemotherapy given for $>12$ months.

\section{Confirmation test of TB patients}

All patients were clinically, radiologically, microbiologically and histologically confirmed cases. IS6110 due to its high numerical and positional polymorphism has become a widely used marker in the epidemiological studies. All TB positive cases were reconfirmed by PCR with IS6110 primers (data not shown).

\section{Drug susceptibility test (DST)}

DST was performed following the standard 1\% proportion method (taking standard critical drug concentration) to identify MTB types. The phenotypic resistance of all isolates was determined at baseline. First line anti-TB drugs were added to reach critical concentrations as following: RIF, $1.0 \mu \mathrm{g} / \mathrm{ml}$; INH, $0.2 \mu \mathrm{g} / \mathrm{ml}$; Streptomycin (SM), $4.0 \mu \mathrm{g} / \mathrm{ml}$ and EMB, $2.0 \mu \mathrm{g} / \mathrm{ml}$. For second line anti-TB drugs, the drugs and concentrations as per the standard critical concentrations, i.e. Kanamycin (KM), $30 \mu \mathrm{g} / \mathrm{ml}$; Ofloxacin (OFX), $2.0 \mu \mathrm{g} / \mathrm{ml}$ and Amikacin (AM) $40 \mu \mathrm{g} / \mathrm{ml}$ [13]. History rel- 
evant to TB such as time and duration, outcome of patients was recorded in a predesigned data sheet [14].

The media were examined at $48 \mathrm{~h}$ and then weekly to check for the growth of mycobacteria [15]. The results were read for the first time on 28th day by counting the colonies grown on different slants and the proportion of resistant bacilli was calculated by comparing counts on drug free and drug containing LJ medium represented as percentages. The control media must show growth of at least 50 to 150 colonies. MTB H37Rv was used as a control strain. Any strain with $1 \%$ (the critical proportion) of bacilli resistant to any of the four drugs-RIF, INH, EMB and SM were classified as resistant to that drug. Drug susceptibility was tested every 2-3 months. For all patients, treatment regimens were adjusted on the basis of the results of these evaluations at monthly intervals.

\section{Ethical issues and approval}

Ethical approval for the study was granted by Research cell, King George's Medical University, UP, Lucknow Institutional Ethics Committee (IEC) with approval no. 576/Ethics/R.cell-16 dated 26/10/2016 (Ref Code: 81st ECM II-B/PI). Informed consent was obtained from all patients enrolled for the study.

\section{Results}

Demographic profile of patients

Out of 218 samples collected, a total of 207 sputum smear positive PTB patients were selected. Of these 218 smear positive cases, culture for mycobacteria were positive in 207 cases, contamination was observed in 5 and no mycobacterial growth was seen in 6 cases (Table 1). The study was carried out on 207 participants (Tables 1 and 2). Their demographic profiles and details are summarized in Table 3. Among 207 cases 120 were male and remaining 87 were female. Males were predominant in all age groups in terms of absolute numbers. All of them were in the age group of 19-60 years. Majority of the patients came from urban area. Out of total 207, 129 belonged to urban areas and rest 78 were from rural areas. On the basis of occupation the patients were categorized into manual workers (150), non -manual workers (35) and house wives (22). Their percentage for living in kuchcha and pucca house was found be $24(11.59 \%)$ and $183(83.40 \%)$ respectively. Of the 207 cases, 118 were in low income group and 89 from middle-income group (Table 3).

None of the patients had EPTB, Diabetes mellitus or any other co-morbidity. During the study period, 61 patients were those who had previously had TB. Most patients excreted large numbers of bacilli in sputum (median score, 2.0). At the time that TB was originally diagnosed, all patients were treated with WHO guidelines

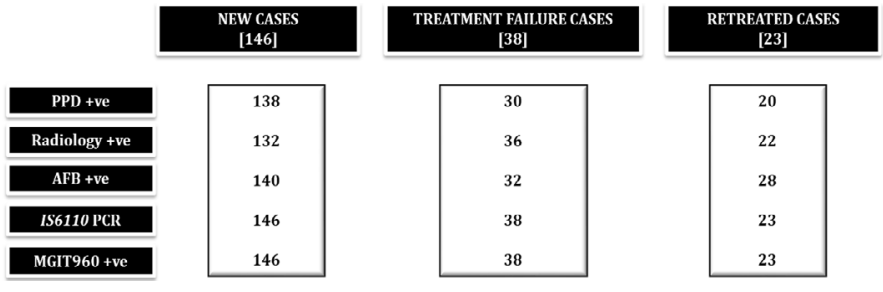

Table 1: Categories of selected TB patients $(n=207)$ and their confirmation by different methods.

DST confirmed samples were categorised into three and there profile was generated using different confirmatory tests (PPD,

Radiology, AFB staining, IS6110 PCR, MGIT960). N=207.

\begin{tabular}{|c|c|c|c|}
\hline SN & Variables & $\begin{array}{l}\text { No. of patients } \\
(\mathrm{N}=207)\end{array}$ & $\begin{array}{c}\text { Percentage } \\
\text { (\%) }\end{array}$ \\
\hline \multirow[t]{4}{*}{1.} & Gender M:F & 120: 87 & 58: 42 \\
\hline & Residence & & \\
\hline & Urban area & 129 & 62.32 \\
\hline & Rural area & 78 & 37.68 \\
\hline \multirow[t]{4}{*}{2.} & Occupation & & \\
\hline & Manual workers & 150 & 72.46 \\
\hline & Non-Manual workers & 35 & 16.91 \\
\hline & Hivuse vives & 22 & 10.63 \\
\hline \multirow[t]{3}{*}{3.} & Type of House & & \\
\hline & Kachcha House & 24 & 11.59 \\
\hline & Pucca House & 183 & 88.41 \\
\hline \multirow[t]{3}{*}{4.} & Socioeconomic Status & & \\
\hline & Low income group & 118 & 57.00 \\
\hline & Middle-income group & 89 & 42.99 \\
\hline \multirow[t]{5}{*}{5.} & Smoking habit & & \\
\hline & Cigarette & 68 & 32.85 \\
\hline & Drug addiction & 55 & 26.57 \\
\hline & $\begin{array}{l}\text { Alcohol } \\
\text { Biofuels }\end{array}$ & 174 & 84.05 \\
\hline & & 19 & 09.17 \\
\hline 6. & Co-morbidity & None & --- \\
\hline \multirow[t]{4}{*}{7.} & Previous anti-TB drug & & \\
\hline & New cases & 146 & 70.53 \\
\hline & Retreated cases & 23 & 11.11 \\
\hline & $11 \mathrm{e}$ & 38 & 18.35 \\
\hline
\end{tabular}

Table 2: Demographic profile of selected patients.

History of patients recruited for the study was taken in methodical manner. This was considered to generate an epidemiological profile of patients. The profile demarcated the patients according to their socio-economic status, earlier exposure with TB, occupation and gender. 


\begin{tabular}{|c|c|c|c|c|}
\hline SN & Drugs & $\begin{array}{c}* \text { New patients } \% \\
\mathbf{n}=148\end{array}$ & $\begin{array}{c}* * \text { Previously treated } \\
\text { patients } \% ; n=19\end{array}$ & $\begin{array}{l}\text { **Treatment failure } \% \\
\mathbf{n}=40\end{array}$ \\
\hline & \multicolumn{4}{|c|}{$1^{\text {st }}$ line drug resistance (FLD) } \\
\hline 1. & Isoniazid (INH) & $38(25.67)$ & $05(26.31)$ & $18(45.00)$ \\
\hline 2. & Rifampicin (RIF) & $50(33.78)$ & $06(31.54)$ & $20(50.0)$ \\
\hline 3. & Streptomycin (SM) & $35(23.64)$ & $05(26.31)$ & $02(05.0)$ \\
\hline 4. & Ethambutol (EMB) & $25(16.89)$ & $04(21.05)$ & None \\
\hline \multirow[t]{2}{*}{5.} & Pyrazinamide (PZA) & Not done & Not done & Not done \\
\hline & \multicolumn{4}{|c|}{$2^{\text {nd }}$ line drug resistance (SLD) injectable } \\
\hline 6. & Kanamycin (KM) & $01(0.67)$ & 0.00 & 0.00 \\
\hline \multirow[t]{2}{*}{7.} & Amikacin (AM) & $04(2.70)$ & $02(10.5)$ & $06(15.0)$ \\
\hline & \multicolumn{4}{|c|}{$2^{\text {nd }}$ line drug resistance (fluoroquinolones) } \\
\hline 8. & Ofloxacin (OFX) & $02(1.35)$ & $04(21.05)$ & $01(02.5)$ \\
\hline 9. & Levofloxacin (LFX) & Not Done & Not Done & Not Done \\
\hline
\end{tabular}

Table 3: Individual drug resistance patterns in selected patients.

*New TB patients: Newly diagnosed sputum smear-positive pulmonary TB (PTB) patients without a history of prior treatment for TB, or a history of treatment for less than 30 days.

** Previously treated/Treatment failure: Diagnosed patients with sputum smear-positive pulmonary TB with a history of prior anti-TB therapy. This may include relapse, treatment after default and treatment after failure, or other patients who have claimed to have anti-TB treatment for more than 30 days.

for varying lengths of time [15]. Once MDR-TB/ FQ resistance was diagnosed, the patients were switched to treatment regimens tailored to phenotypic drug-susceptibility profile of their isolates. At the time of start of the study, therapy was again adjusted according to phenotypic drug susceptibility, treatment history, and the side effects profile (Table 3 ).

\section{Phenotypic profiling of Mtb}

All 207 isolates displayed phenotypic resistance to RIF and taken together the isolates from all patients were highly resistant to many of the potent first line agents. Figure 2 shows pattern of drug resistance in the included patients. The highest value with any resistance was found in 152 cases followed by MDR cases, 43 . 23 patients were found to be drug susceptible. Table 3 shows the drug susceptibility and resistance pattern of MTB strains of PTB clinical isolates to 4 first line ATT drugs. Most of the strains were resistant to one or more ATT drugs. In this group of patients the highest mono drug resistance was found in RIF either alone or in combination with other drugs (with two drugs, RIF and INH; with three drugs, RIF+INH+SM and with 4 drugs, RIF+INH+EMB+SM) (Figure 3). The rate of prevalence of drug resistance to the first line drugs and second line in new, retreated and treatment failure cases is summarized in Table 3. The highest resistance was found with RIF in 20 treatment failure patients (50.0\%) which was followed by $33.78 \%$ in new cases. The second highest resistance
(45.0\%) was again in treatment failure patients with INH followed by $26.31 \%$ in retreated cases and minimum $25.68 \%$ was with newly diagnosed TB patients. SM resistance was highest with retreated cases, $26.31 \% ; 23.64 \%$ in new cases and only $5 \%$ in treatment failure cases respectively. The EMB resistance was highest $(21.05 \%)$ in retreated cases followed by new cases, $16.89 \%$. In our study 43 $(17.14 \%)$ isolates were identified as MDR; the other isolates were resistant to all the 3 and 4 drugs tested (Table 3).

Drug resistance patterns of MTB strains collected from newly diagnosed sputum smear-positive TB patients

Resistance patterns to individual first-line drugs tested indicated highest resistance to RIF (33.78\%) followed by resistance for INH in 38 patients (25.67\%), SM (23.64\%) and EMB (16.89\%) as shown in Table 3. Resistance patterns to individual second line drugs tested indicated highest resistance to AMK (2.70\%), followed by resistance for OFX (1.35\%), KAN (0.67 \%) as shown table 3 .

Individual drug resistance pattern among previously treated and treatment failure TB patients

Among the 19 previously treated TB patients subjected to DST, none were susceptible to all drugs tested, while all (100\%) showed resistance to any drug. MDR-TB was detected in 43 (20.77\%) patients (Figure 3). Mono drug resistance to rifampicin was observed in 6 patients (31.54\%) among the previously treated and in 20 (50 
$\%$ ) among treatment failure cases. Resistance patterns to individual first-line drugs tested indicated highest resistance to RIF (in retreated cases $31.54 \%$ and in treatment failure cases 50\%) followed by resistance for INH (in retreated cases $26.31 \%$ and in treatment failure cases $45 \%$ ) and SM (in retreated cases $26.31 \%$ and in treatment failure cases 5\%) as shown in Table 3.

Resistance patterns to individual second line drugs tested indicated highest resistance to OFX (in retreated cases $21.5 \%$ and in treatment failure cases it was $2.5 \%$ ) followed by resistance for AMK (in retreated cases $10.5 \%$ and in treatment failure cases $15 \%$ ) and KAN (any 1.01\%, mono $0.0 \%$ ) as shown in Table 3. Injectable second line drugs (KAN and AMK) exhibited similarly low frequencies of resistance respectively. This indicates that second line injectable drugs have no superiority over each other. MDR-TB was detected in $43(20.77 \%)$ of the TB patients tested as shown in Figure 2 and Figure 3. RIF resistance was always accompanied by INH resistance. Poly drug resistance (PDR) was observed in 26 cases (RIF + SM, 21 cases and RIF + EMB, 5 cases)

\section{Discussion}

TB is one of the oldest recorded, highly prevalent microbial human infections. Its late diagnosis and treatment increases the risk of disease dissemination and decreases the survival of patients [16-18]. The diagnosis of PTB is based on the identification of the bacillus by microscopic examination of sputum smear or by culture (Figure 1, Table 1). India is one of the highest burden countries for TB and DR-TB. However, the epidemiology of DR-TB in India has never been studied nationally. The journey from compound to drug to resistance is very long. In 1951, INH replaced SM for which resistance and toxicity had already raised problems in TB treatment. The first emergence of INH resistance in MTB clinically appeared in 1952. It has become essential to measure the DR-TB burden and to guide development of evidence-based strategies to combat drug resistance in India [19-22].

Our study was conducted on 207 patients, presented by smear positive PTB and proved to be culture positive for TB using LJ medium; searching for the prevalence of anti-mycobacterial drug resistance and the pattern of drug resistance (Figure 2). The drug susceptibility testing was carried also on quinolones and aminoglycosides (presented by OFX, LFX, and AMK), to study their efficacy on the DR strains and to determine the frequency of emergence of FQ-resistant strains (Table 3). Drug susceptibility testing was done using the proportion method. The proportion method is the most commonly used method worldwide. It allows the precise determination of the proportion of resistant mutants to a certain drug [11].

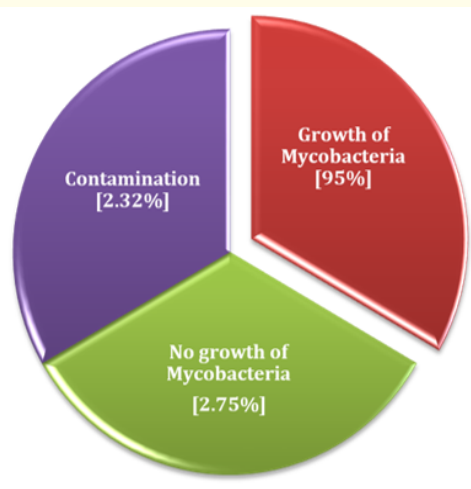

Figure 1: Results of Culture of Smear (AFB) positive sputum specimen $(\mathrm{n}=218)$.

Of the total 218 patients recruited in the study, when the bacteria were grown in LJ slants, 207 were finally taken up for the study. All 218 patients were AFB positive via microscopy. When cultured 207/218 samples were culture positive for MTB.

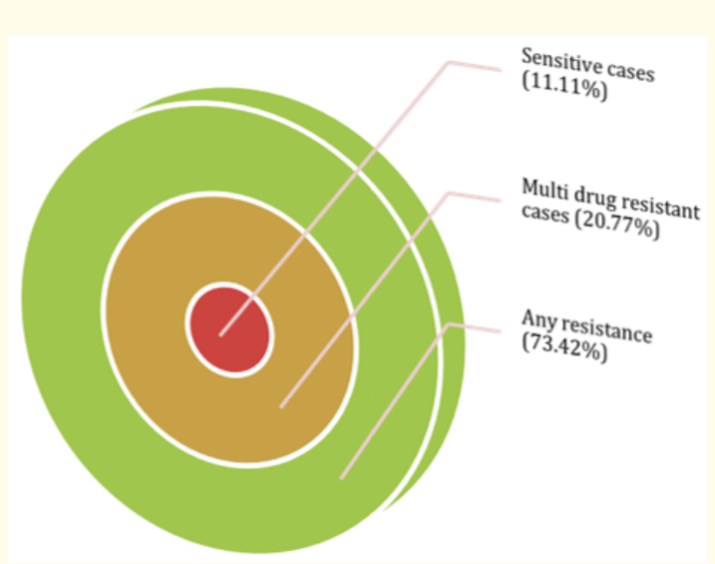

Figure 2: Pattern of drug resistance in selected patients.

More than two third of the samples were resistant to at least one of the drugs used in ATT. And almost one third were resistant to both RIF+INH combined leading to multi drug resistance. $\mathrm{N}=207$

The efficiency of current TB control program in any country is assayed by drug resistance pattern. In 1993, the NTP in India was revised and strengthened in the form of Revised National Tuberculosis Control Programme (RNTCP). Like HIV-AIDS, threat perception due to occurrence of multidrug resistance has assumed considerable gravity in constructing the epidemic situation analysis and appropriate intervention $[10,23,24]$. In this study drug resistance of MTB to at least one drug were found in all selected cases 
(Table 3, Figure 3). This situation is highly alarming. A review of the Indian situation from the TRC, Chennai, has concluded that the magnitude of the drug resistance problem is principally due to acquired resistance (replaced in recent times by the term drug resistance among previously treated cases) [25]. In New Delhi, a similar extent of acquired drug resistance was reported. It was considered 53\% that initial drug resistance in India (freshly defined as, drug resistance among new cases) could be at a lower order than similarly placed countries globally, as distinct from the acquired drug resistance situation given above. There could be $5-10 \%$ resistance to INH. This could be reflecting the primary drug resistance problem in the Indian context [26,27]. In this study 69 isolates (33.33\%) resistant to two or more of the tested drug were identified. This is comparable to what has been reported in the neighboring countries, with resistance to INH and RIF being more common than resistance to EMB (Figure 3). Resistance to RIF is increasing because of widespread application that results in selection of resistant mutants, and is seen in cases non-compliant with TB treatment. In this context, resistance to RIF can be assumed to be a surrogate marker for MDR-TB. Phenotypic susceptibility testing for Pyrazinamide (PZA) was not performed, because the results of this test can be difficult to reproduce and may not correlate well with drug susceptibility in vivo $[18,20,24]$.

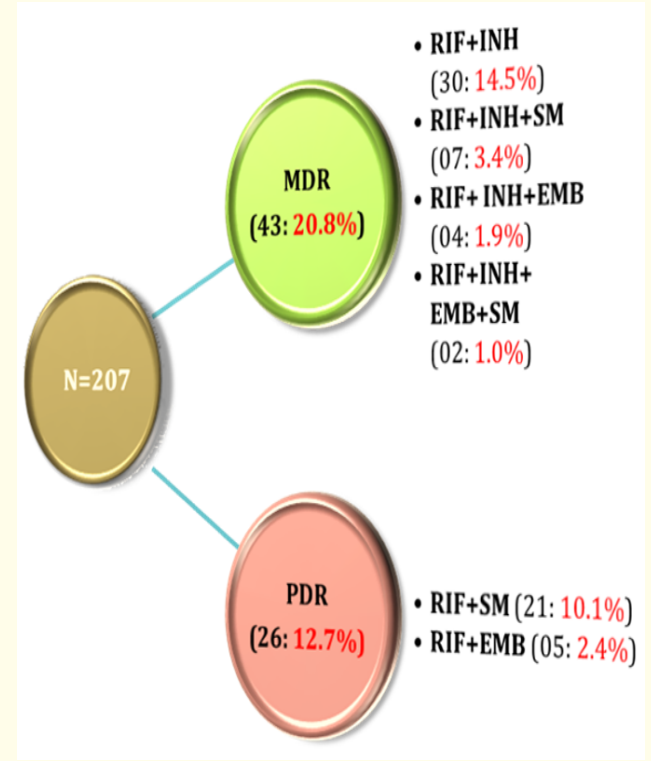

Figure 3: Multi drug resistance pattern of clinical isolates to anti tuberculosis drugs.

Poly drug resistance (PDR) was also observed in $12.7 \%$ of cases, wherein the bacteria was resistant to either RIF or INH along with another first line drug. We observed PDR with RIF in the 26 cases.
INH and RIF resistance in MTB complex (MTBC) isolates are mainly based on mutations in a limited number of genes. However, mutation frequencies vary in different mycobacterial populations. In 1992, Stewart Cole applied genetics to identify the first gene responsible for INH resistance in MTB clinical isolates. This information soon translated into genotyping for antibiotic resistance in clinical settings. Today, with the development of rapid whole genome sequencing, we are at the helm of understanding at the global genomic level how bacteria retain their fitness despite their ability to mutate and develop drug resistance [28-31]. It's been over 100 years in our battle against TB but mechanisms underlying the interactions of the bacillus and its host are far from understood. So clinically, multi-drug resistance may be an outcome of several mono-drug resistant strains in addition to being a case of MDR at the strain level. Given that drug resistance exists against all current TB drugs, the need for new drugs has been a focus of many research quests ranging from target based and whole cell screening approaches, to the identification and validation of new targets. Basic research has provided and continues to provide new solutions, awareness, and novel paradigms for treatment.

\section{Conclusion}

To meet the challenge of MDR-TB and XDR-TB worldwide, huge monetary resource instillation and extensive human resource development are required for the prevention and management of these formidable drug resistance scenarios. Among the response priorities, rapid detection of anti-tuberculosis drug resistance, use of appropriate regimens for treatment, and new drug development are of paramount importance. Recent advances in high throughput DNA sequencing will allow whole genome sequencing of unique drug-resistant strains to be performed at a much more rapid speed and significantly less cost, which will facilitate the identification of new and unknown mechanisms of drug resistance and ultimately lead to more efficient detection of drug resistance. Improved understanding of the mechanisms of drug resistance in MTB would be conducive to the accelerated development of these new strategies for control of DR-TB. However, strengthening of the current TB control programs globally should be a continuous process. Adequate monitoring of drug resistance, especially MDR/XDR-TB in new patients and its transmission, molecular characterization of the drug-resistant strains, and analysis of patients' immune status and genetic susceptibility are also needed to address the problem of the fitness, virulence and transmissibility of DR-MTB strains.

\section{Acknowledgement}

We acknowledge the Vice Chancellor, KGMU for his support and encouragement. We also acknowledge all the patients who provided us the samples for research purpose. 


\section{Conflict of Interest}

None declared.

\section{Bibliography}

1. Zignol M., et al. "Global Incidence of Multidrug-Resistant Tuberculosis". (2006): 479-485.

2. WHO. WHO Global TB 2018 Report (2018).

3. Kant S., et al. "Multi-drug resistant tuberculosis: An iatrogenic problem". BioScience Trends 4 (2010): 48-55.

4. Eldholm V., et al. "Four decades of transmission of a multidrug-resistant Mycobacterium tuberculosis outbreak strain". Nature Communications 6 (2015): 1-9.

5. Nguyen L. "Antibiotic resistance mechanisms in Mtb: an update". Archives of Toxicology 90 (2017): 1585-1604.

6. Behr MA., et al. "Revisiting the timetable of tuberculosis". BMJ 362 (2018).

7. Zhang Y. "The Magic Bullets and Tuberculosis Drug Targets". 45 (2005): 529-564.

8. Liu Q., et al. "Analysis of risk factors for drug resistance in tuberculosis patients". 11 (2019): 1-5.

9. Chang K., et al. A systematic review of rapid drug susceptibility tests for multidrug-resistant tuberculosis using rifampin resistance as. Expert Opinion on Medical Diagnostics (2009): 99-122.

10. Elmi OS., et al. "Multidrug-resistant tuberculosis and risk factors associated with its development: A retrospective study". The Journal of Infection in Developing Countries 9 (2015): 1076-1085.

11. Heifets L. "Conventional methods for antimicrobial susceptibility testing of Mycobacterium tuberculosis". (2000): 133143.

12. Sharma M., et al. “Comparison of modified Petroff's and N-acetyl-L-cysteine-sodium hydroxide methods for sputum decontamination in tertiary care hospital in India". Medical Journal of Dr. DY Patil Vidyapeeth (2012): 97-100.

13. Yuan X., et al. Molecular Characterization of Multidrug- and Extensively Drug- Resistant Mycobacterium tuberculosis Strains in Jiangxi, China". Journal of Clinical Microbiology 50 (2012): 2404-2413.

14. Kent PT and Kublica GP. "Public health mycobacteriology. A guide for the level III laboratory". Atlanta, Georgia: US Department of Health and Human Services, Centers for Disease Control (1985): 96-103.
15. Falzon D., et al. HHS Public Access. 42 (2015): 156-168.

16. Daniel TM. "The history of tuberculosis". Respiratory Medicine 100 (2006): 1862-1870.

17. Kolyva A and Karakousis P. Old and new TB drugs: Mechanisms of action and resistance. Underst Tuberc - New Approaches to Fight Against Drug Resist (2012): 210-232.

18. Murray JF., et al. "Treatment of tuberculosis: A historical perspective". Annals of the American Thoracic Society 12 (2015a): 1749-1759.

19. Yee D., et al. "Incidence of Serious Side Effects from First-Line Antituberculosis Drugs among Patients Treated for Active Tuberculosis". American Journal of Respiratory and Critical Care Medicine 167 (2003): 1472-1477.

20. Campbell PJ., et al. "Molecular Detection of Mutations Associated with First- and Second-Line Drug Resistance Compared with Conventional Drug Susceptibility Testing of Mycobacterium tuberculosis". Antimicrobial Agents and Chemotherapy 55 (2011): 2032-2041.

21. Murray JF., et al. "Treatment of tuberculosis: A historical perspective". Annals of the American Thoracic Society 12 (2015b): 1749-1759.

22. Furin J., et al. Tuberculosis. Lancet 393 (2019): 1642-1656.

23. Merker M., et al. "Evolutionary history and global spread of the Mycobacterium tuberculosis Beijing lineage". Nature Genetics 47 (2015): 242-249.

24. Ramirez-Busby SM and Valafar F. "Systematic Review of Mutations in Pyrazinamidase Associated with Pyrazinamide Resistance in Mycobacterium tuberculosis Clinical Isolates". Antimicrobial Agents and Chemotherapy 59 (2015): 5267-77.

25. Selvakumar N., et al. "High Rates of Ofloxacin Resistance in Mycobacterium tuberculosis among Both New and Previously Treated Patients in Tamil Nadu, South India". Plos one (2015): $1-9$.

26. Narayanan S., et al. "Molecular Epidemiology of Tuberculosis in a Rural Area of High Prevalence in South India: Implications for Disease Control and Prevention". Journal of Clinical Microbiology 40 (2002): 4785-4788.

27. Torres JN., et al. "Novel katG mutations causing isoniazid resistance in clinical M. Tuberculosis isolates". Emerging Microbes and Infections 4 (2015): e42.

28. Baek SH., et al. "Metabolic regulation of mycobacterial growth and antibiotic sensitivity". PLoS Biology (2011). 
29. Comas I., et al. "Whole-genome sequencing of rifampicinresistant Mycobacterium tuberculosis strains identifies compensatory mutations in RNA polymerase genes". Nature Genetics 44 (2012): 106-110.

30. Dookie N., et al. "Evolution of drug resistance in Mycobacterium tuberculosis: a review on the molecular determinants of resistance and implications for personalized care". Journal of Antimicrobial Chemotherapy (2018): 1138-1151.

31. Oppong YEA., et al. Genome-wide analysis of Mycobacterium tuberculosis polymorphisms reveals lineage- specific associations with drug resistance. BMC genomics 252 (2019): 1-15.

\section{Volume 2 Issue 10 October 2019}

(C) All rights are reserved by Surya Kant, et al. 